
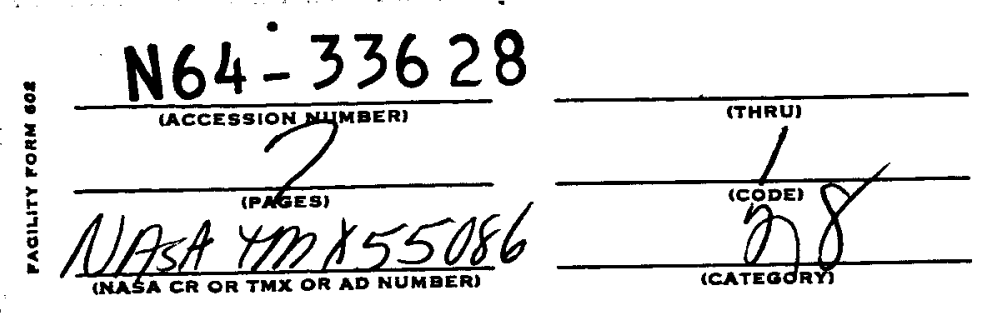

\title{
THE DISTRIBUTION IN HELIOGRAPHIC LONGITUDE OF FLARES WHICH PRODUCE ENERGETIC SOLAR PARTICLES
}

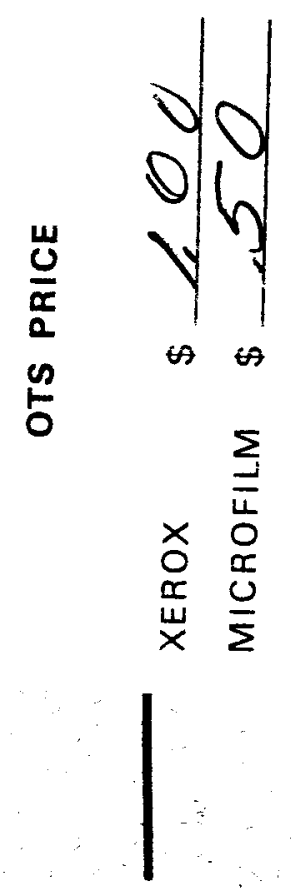

\author{
BY \\ DONALD E. GUSS
}

NASA

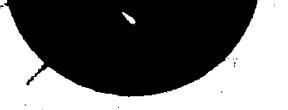

1

JULY 1964

\section{GODDARD SPACE FLIGHT CENTER \\ GREENBELT, MARYLAND}

Goddard Energetic Particles Preprint Series 
THE DISTRIBUTION IN HELIOGRAPHIC LONGITUDE OF FLARES WHICH PRODIIE ENERGETIC SOLAR PARTICLES

\author{
Donald E. Guss \\ NASA-Goddard Space Flight Center, Greenbelt, Md.
}

A number of evidences have been found in the past which point to the longitudinal persistence of solar phenomena. The life of active regions on the sun extends over several solar rotations and centers of sun spot formation exist which seem to outlive individual spot groups. 1,2 Also there are 27 day recurrences of geomagnetic disturbances $^{3}$ and neutron monitor counting rate data ${ }^{4}$, which sometimes last for as long as ten solar rotations. More recently Bryant et al. ${ }^{5}$ and simpson 6 have found twenty-seven day recurrences in the enhancement of low energy proton intensity in free space as measured on satellites. It is the purpose of this letter to point out a similar longitudinal persistence in the occurrence of solar flares which result in solar particle events. In particular, flares from a single $10^{\circ}$ interval in heliographic longitude caused most of the large solar particle events over the last solar cycle. This indicates the existence of a center for the formation of active regions which has persisted for more than 73 solar rotations.

Figure 1 shows the heliographic longitude for flares which produced solar particle events between 16 January 1955 and 23 October 1962 during the last solar cycle. The list of 56 events used is from that compiled by Malitson ${ }^{7}$ plus the 23 October 1962 event. The series of flares between $80^{\circ}$ and $90^{\circ}$ in Figure 1 produced the largest particle events of the last 
solar cycle, the event of 23 February 1956, the multiple events of July 1959, the multiple events of November 1960, and the multiple events of July 1961. Using the integrated particle intensities calculated by Malitson and Webber ${ }^{8}$, these events contributed about $75 \%$ of the integrated particle intensity with energy greater than $30 \mathrm{Mev}$ and about $90 \%$ of that with kinetic energy $>100 \mathrm{Mev}$ detected at the earth during the last solar cycle. In addition, of the ten solar particle events which produced neutron monitor increases, indicating the presence of significant numbers of particles with kinetic energy greater than about $500 \mathrm{Mev}$, seven are in this narrow longitude region.

Trotter and Roberts ${ }^{9}$ have noted that the vicinity of the sun where the 23 February 1956 flare occurred had had a prior history of active regions lasting for several rotations, dying out, and returning in new bursts of activity. Hence, this center of activity existed prior to the 23 February 1956 event and persisted beyond the event of 20 July 1961 , a period of more than 5.5 years. Babcack ${ }^{10}$ in his theory of the sun's field proposes a mechanism to explain the longitudinal persistence whereby new active regions are likely to arise near old regions. However, it is not clear whether this mechanism is sufficient to explain an active site of such long duration. The remainder of the events during the last solar cycle also fall into longitude bands, but not so sharply defined. The events between $240^{\circ}$ and $280^{\circ}$ with one exception occurred between 20 January 1957 and 23 March 1958 indicating the existence of an active site which lasted for more than a year. The events between $210^{\circ}$ and $220^{\circ}$ are those of March through September 1960 two of which caused ground level neutron monitor increases. The interval 
between $160^{\circ}$ and $190^{\circ}$ contains events which occurred between 9 August 1957 and 10 May 1959. The large event is that of 10 May 1959. The interval between $110^{\circ}$ and $140^{\circ}$ includes events from 6 June 1958 to 22 August 1958 and the two small events of 10 September and 28 September 1961 .

Finally, there is a dearth of events in the longitude interval between $280^{\circ}$ and $80^{\circ}$. Of the 26 larger particle events during the last solar cycle only two, the events of 31 August 1956 and 7 July 1958 , resulted from flares in this $160^{\circ}$ interval.

In conclusion, flares which produce energetic particles arise predominantly in narrow longitudinal regions which outlive visible active regions. This points to the existence of long lived active centers beneath the photosphere which periodically manifest themselves on the surface as active regions which produce flares that accelerate solar particles. A single well defined longitude region was responsible for the most intense particle events of the last solar cycle. The fact that this region can be compressed into a ten degree band of longitude with a suitable choice of the period of solar rotation would indicate that this site rotated at constant rate as opposed to the variable rotation of the photosphere. The shorter lived regions, responsible for the smaller events, in general show a wider spread in longitude which could indicate a drift of the producing centers. A study of the meridional distribution of a larger body of data such as solar flares would perhaps result in a further definition of the active centers. 


\section{References}

1. K. 0. Kiepenheuer, The Sun edited by G. P. Kuiper, p. 338, University of Chicago Press (1953).

2. H. M. Losh, Publications of the Observatory of the University of Michigan, ㄱ, 127 (1938).

3. J. Bartels, Terr. Mag. Atm. Elect. 37, 48 (1932).

4. J. A. Simpson, W. Fonger, and L. Wilcox, Phys. Rev. 85, 366 (1952).

5. D. A. Bryant, T. L. Cline, U. D. Desai, and F. B. McDonald, Phys. Rev. Letters 11 , 144 (1963).

6. J. A. Simpson, IMP Symposium, Goddard Space Flight Center (March 1964).

7. H. H. Malitson, NASA Technical Report R-169 edited by F. B. McDonald, p. 109 (1963).

8. H. H. Malitson and W. R. Webber, NASA Technical Report R-169 edited by F. B. McDonald, pp. 12, 13 (1963).

9. D. E. Trotter and W. 0. Roberts, Solar Activity Summary I, High Altitude Observatory, 8 (1956).

10. H. W. Babcock, Astrophys. J. 133, 581 (1961). 


\section{FIGURE CAPTION}

Figure 1: Number of solar particle events vs. heliographic longitude with the central meridian during the 23 February 1956 event set to $0^{\circ}$ and assuming a rotation period of 27.04 days. The particle intensity with kinetic energy $>30 \mathrm{Mev}$ detected at the earth ${ }^{8}$ integrated over the particle event is: $\bigotimes, I \geq 10^{8} \mathrm{p} / \mathrm{cm}^{2} ; \square, I \geq 5 \times 10^{6} \mathrm{p} / \mathrm{cm}^{2} ;$ open squares, $I<5 \times 10^{6} \mathrm{p} / \mathrm{cm}^{2}$. A dot in a square signifies that there was a neutron monitor rate increase, indicating the presence of a significant number of particles with kinetic energy greater than about $500 \mathrm{Mev}$. Light lines are used to separate individual events and heavy lines to separate individual active regions. 


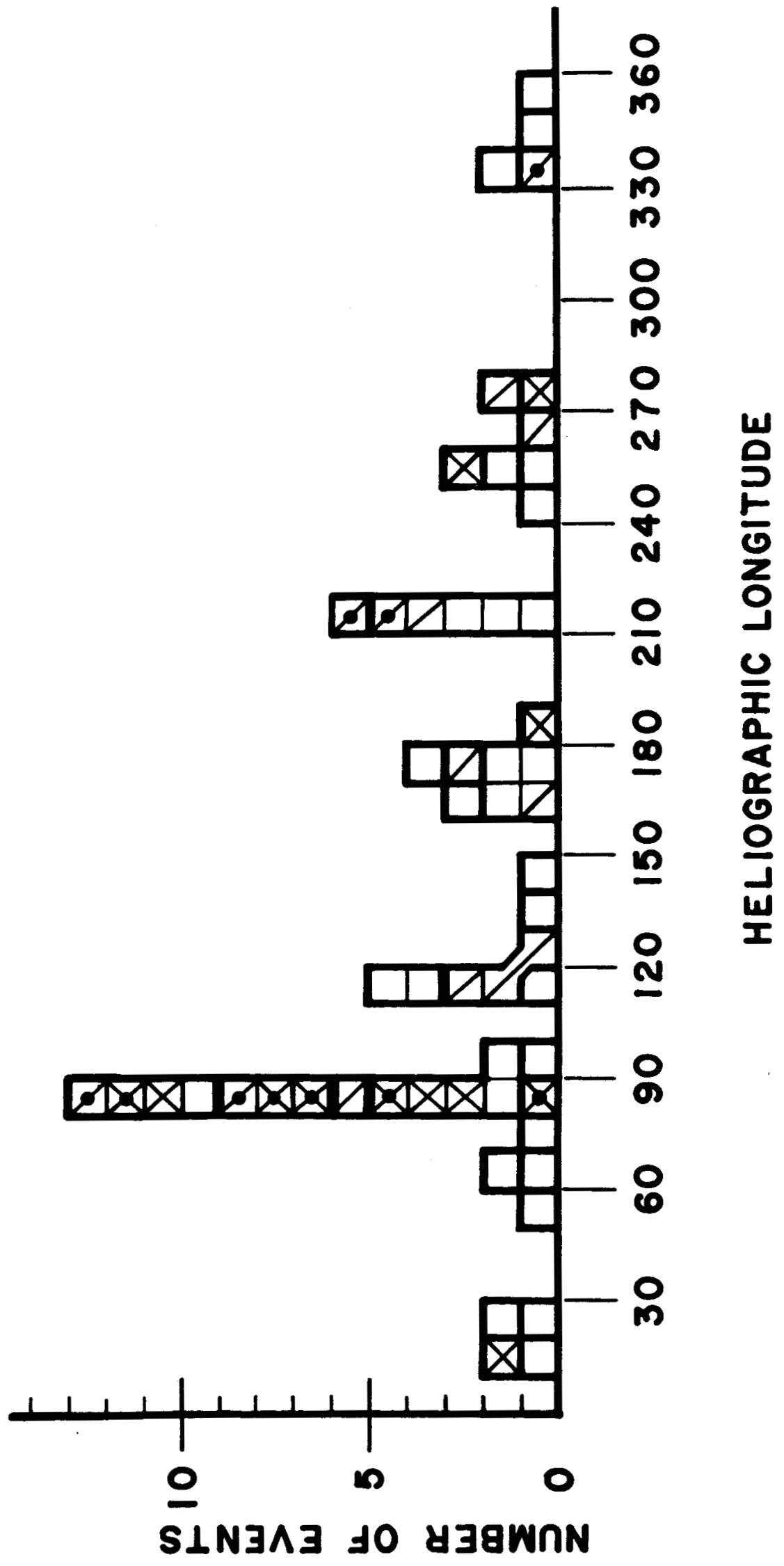

